Ann. Génét. Sél. anim., 1975, 7 (1), 9I-97.

\title{
L'EFFICACITÉ DE LA SÉLECTION POUR LE NOMBRE DE MAMELLES CHEZ LE PORG DE PIÉTRAIN
}

\author{
R. HANSET et J. WIDAR \\ Faculté de Médecine vétérinaire, \\ Cureghem, Bruxelles (Belgique) \\ RÉSUMÉ
}

Les auteurs étudient les effets d'une sélection en faveur du nombre de tétines chez les porcs de Piétrain, pour la période 196I-1971. Le nombre moyen de tétines est passé de 13,18 à 13,59 et le pourcentage de sujets éliminés pour nombre insuffisant de tétines de 6,49 à $\mathrm{I}, 78 \mathrm{p}$. 100. Des phénotypes à $\mathrm{I}_{7}$ et $\mathrm{I} 8$ tétines, nouveaux pour la race, sont apparus en fin de période.

Partant de 1'idée que le nombre de porcelets sevrés peut être limité par le nombre de mamelles de la truie, les Syndicats d'Élevage porcin prescrivent que, pour être tatoué, un porcelet doit avoir au moins douze tétines. Le nombre de porcelets éliminés de l'élevage pour cette raison est fonction du nombre moyen de tétines de la race. Ainsi, en r96r, chez le Porc de Piétrain, où la moyenne raciale était de 13,17, 6,5 p. Ioo des porcelets n'atteignaient pas le nombre requis de douze tétines, alors que chez le Landrace Belge où la moyenne était de $13,99,0,38$ p. Ioo seulement étaient éliminés. (HANSE'T et CAMERLYNCK, I974).

Une sélection s'exerce donc en permanence chez le Porc de Piétrain, à cet égard. Elle est par contre négligeable chez le Porc Landrace belge.

Elle devrait être efficace sachant que l'héritabilité du nombre de tétines, chez le Porc de Piétrain, est de l'ordre de o,46 (HANSET et CAMERLynck, I974).

C'est ce que nous avons cherché à vérifier.

\section{MATÉRIEL ETT MÉTHODES}

Nous avons pu disposer de données récoltées, à l'occasion des opérations de tatouage, par les Syndicats d'Élevage de la Province de Brabant et relatives à des porcelets nés en 1961, 1963, 1965, 1967, 1969 et 1971. Les tailles de ces différents échantillons étaient les suivantes : 1961 : 
4342 porcelets de 560 truies et 56 verrats; I 963 : I 50 I porcelets de 200 truies et 50 verrats; I965: I 508 porcelets de I 96 truies et 49 verrats; I $967:$ I 529 porcelets de I 96 truies et 49 verrats ; I969: I 557 porcelets de 200 truies et 50 verrats ; I97 I : I 632 porcelets de 200 truies et 50 verrats.

Sur chacun de ces échantillons, nous avons pu calculer, moyenne, écart-type, test de symétrie, proportion d'individus ayant moins de douze tétines, héritabilité. Le coefficient d'héritabilité a été estimé selon trois méthodes :

Io à partir des composantes " paternelle et " maternelle " de la variance ;

$2^{\circ}$ par la régression des enfants sur les mères, intra-père ;

$3^{\circ}$ par la régression de la moyenne des descendants sur la moyenne parentale.

\section{RÉSULTATS}

Le tableau $x$ et les figures I et 2 illustrent l'évolution intervenue en ce qui concerne le nombre moyen de tétines et la proportion de sujets ayant moins de douze tétines.

Le nombre moyen de tétines augmente significativement chez les parents, verrats et truies, ainsi que chez leurs descendants des deux sexes. La moyenne des porcelets femelles est systématiquement plus petite que celle des descendants mâles.

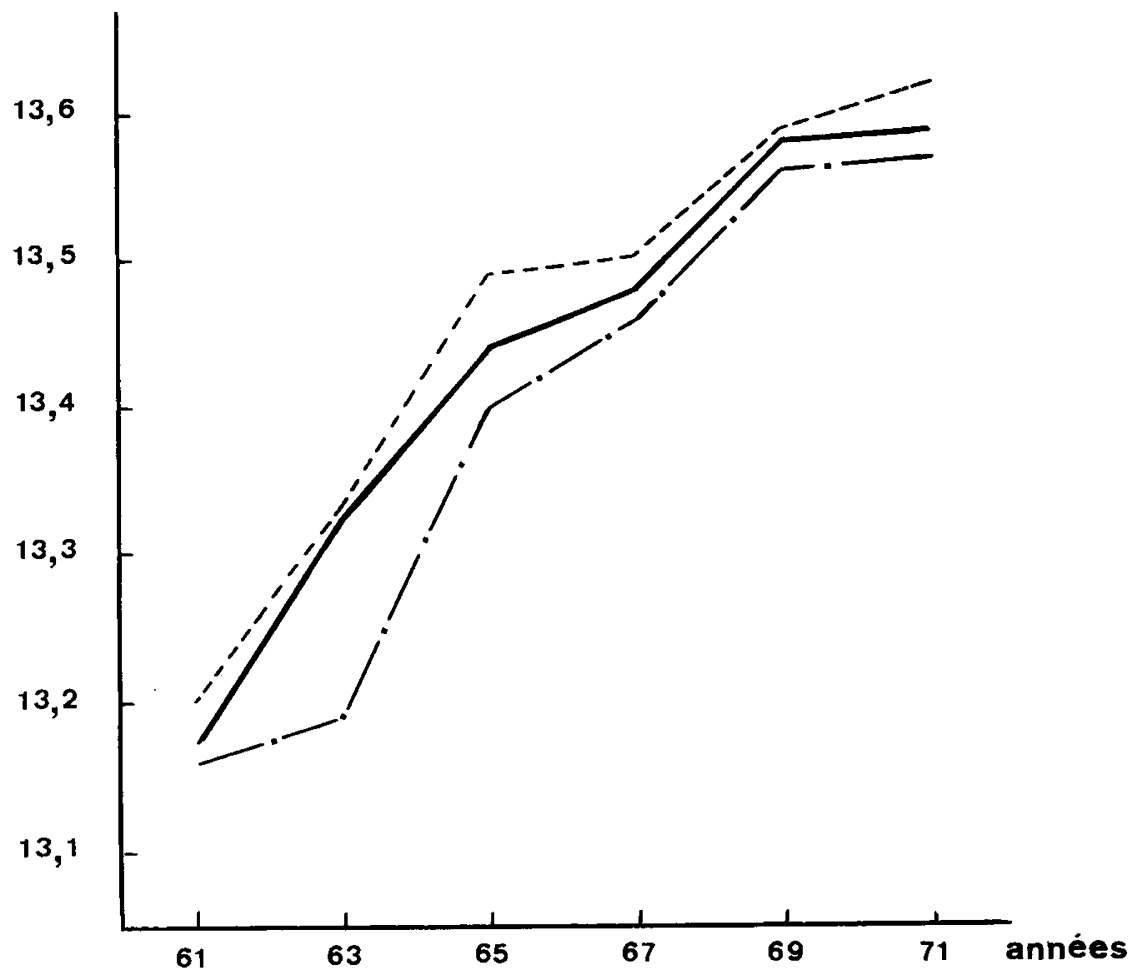

Fig. 1. - Evolution du nombre moyen de tétines au cours de la période 1961-1971

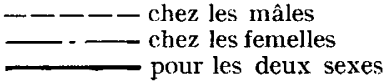




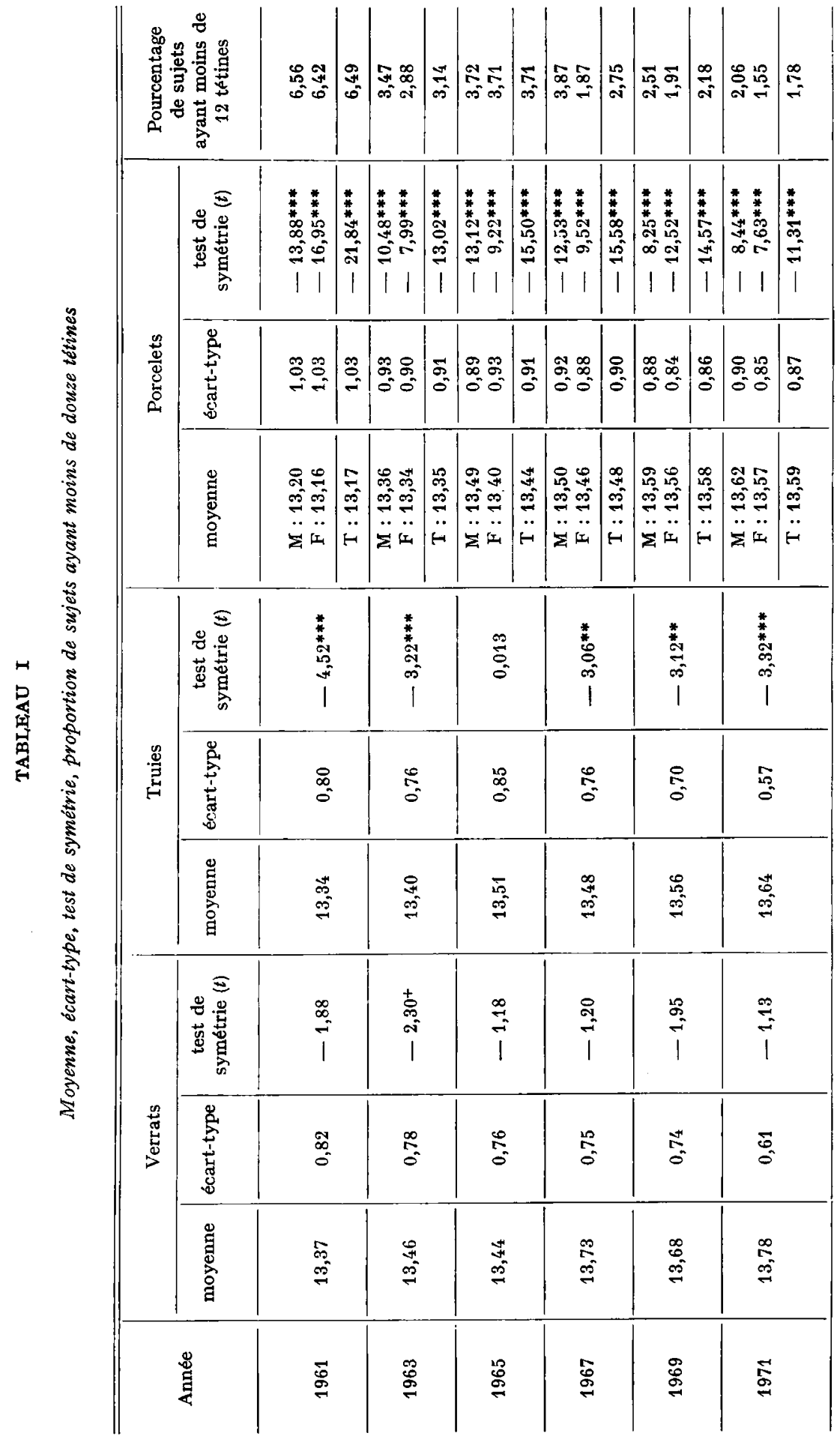

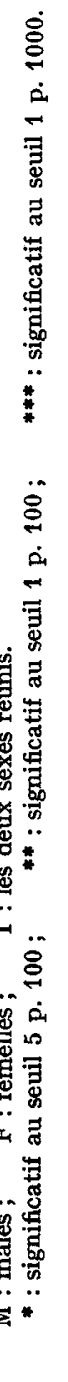


Cette différence est significative pour les années $\mathrm{r} 963$ et 1965 , la plus petite différence significative au seuil $5 \mathrm{p}$. Ioo, étant de l'ordre de 0,07. En outre, la proportion de mâles ayant moins de douze tétines paraît plus élevée. Toutefois, cette différence n'est significative que pour l'année I967 (seuil 5 p. Ioo).

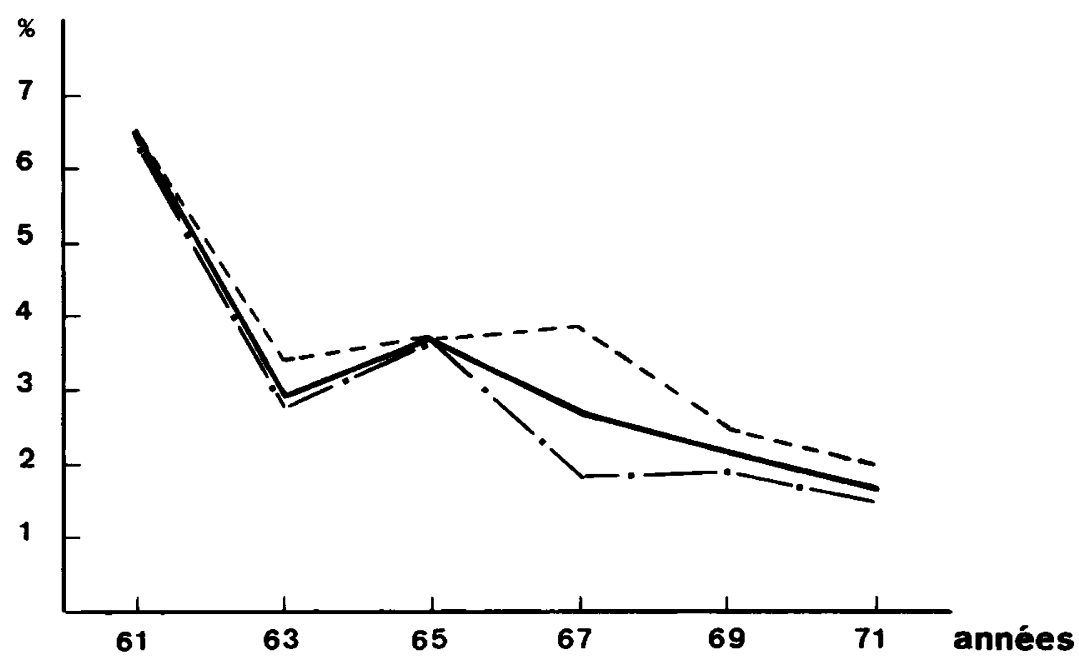

FIG. 2. - Evolution du pourcentage d'individus ayant moins de douze tétines

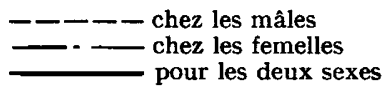

TABLEAU 2

Évolution de la fréquence (en p. Ioo) des différentes classes

\begin{tabular}{c|r|r|r|r|r|r}
\hline \hline & \multicolumn{6}{|c}{ Années } \\
$\begin{array}{c}\text { Nombre } \\
\text { de tétines }\end{array}$ & 1961 & 1963 & 1965 & 1967 & 1969 & 1971 \\
& & & & & & \\
\hline & & & & & & \\
10 & 1,73 & 0,73 & 0,66 & 0,65 & 0,38 & 0,37 \\
11 & 4,77 & 2,41 & 3,05 & 2,09 & 1,80 & 1,41 \\
12 & 18,49 & 14,57 & 10,94 & 12,17 & 9,38 & 9,99 \\
13 & 27,04 & 29,21 & 26,46 & 24,46 & 23,31 & 23,22 \\
14 & 45,53 & 49,87 & 54,71 & 55,27 & 59,09 & 57,90 \\
15 & 2,16 & 2,81 & 3,58 & 5,10 & 5,40 & 6,07 \\
16 & 0,28 & 0,40 & 0,60 & 0,26 & 0,51 & 0,92 \\
17 & & & & & 0,13 & 0,06 \\
18 & & & & & & 0,06 \\
\hline & $100 \%$ & $100 \%$ & $100 \%$ & $100 \%$ & $100 \%$ & $100 \%$ \\
\hline \hline
\end{tabular}

La sélection diminue en intensité dans la mesure où elle est efficace. La distribution du nombre de tétines demeure fortement asymétrique malgré un déplacement de la moyenne vers les valeurs plus élevées. Cette asymétrie qui se traduit par une 
statistique $g_{1}$ négative, d'où un test de $t$ négatif, consiste en un excès de valeurs supérieures à la moyenne.

$\mathrm{Si}$ on examine les fréquences des différentes classes, on constate que la sélection a eu pour résultat de renforcer la fréquence de la classe modale (I4 tétines) et des classes supérieures et aussi de faire apparaître des phénotypes plus extrêmes, c'est-àdire des individus à $\mathrm{I} 7$ et même $\mathrm{I} 8$ tétines (tabl. 2). En I $\mathrm{g} 6 \mathrm{I}$, sur un échantillon de 4342 porcelets, le phénotype à $\mathrm{I} 6$ tétines était le phénotype le plus extrême.

Les différentes estimations du coefficient d'héritabilité sont rassemblées au tableau 3. Comme le laisse prévoir leur écart-type, ces estimations fluctuent fortement d'un échantillon à l'autre, d'une méthode à l'autre.

TABLEAU 3

Héritabilité du nombre de tétines chez le Porc de Piétrain

\begin{tabular}{|c|c|c|c|c|c|}
\hline \multirow{2}{*}{ Années } & \multicolumn{3}{|c|}{ Analyse de variance hiérarchique } & \multirow{2}{*}{$\begin{array}{l}\text { Régression } \\
\text { sur la mère, } \\
\text { intra-père }\end{array}$} & \multirow{2}{*}{$\begin{array}{l}\text { Régression sur } \\
\text { moy. parentale }\end{array}$} \\
\hline & $h_{\mathbf{P}}^{2}$ & $h_{\mathrm{M}}^{2}$ & $h_{\mathrm{P}+\mathrm{M}}^{2}$ & & \\
\hline 1961 & $0,428 \pm 0,99$ & $0,566 \pm 0,06$ & $0,497 \pm 0,056$ & $0,352 \pm 0,056$ & $0,444 \pm 0,038$ \\
\hline 1963 & $0,392 \pm 0,116$ & $0,280 \pm 0,086$ & $0,336 \pm 0,065$ & $0,379 \pm 0,084$ & $0,275 \pm 0,056$ \\
\hline 1965 & $0,232 \pm 0,094$ & $0,430 \pm 0,102$ & $0,331 \pm 0,059$ & $0,216 \pm 0,082$ & $0,182 \pm 0,05$ \\
\hline 1967 & $0,314 \pm 0,121$ & $0,673 \pm 0,124$ & $0,493 \pm 0,074$ & $0,366 \pm 0,100$ & $0,379 \pm 0,064$ \\
\hline 1969 & $0,205 \pm 0,098$ & $0,655 \pm 0,122$ & $0,430 \pm 0,065$ & $0,462 \pm 0,097$ & $0,397 \pm 0,065$ \\
\hline 1971 & $0,143 \pm 0,096$ & $0,852 \pm 0,141$ & $0,497 \pm 0,069$ & $0,324 \pm 0,086$ & $0,233 \pm 0,064$ \\
\hline $\begin{array}{c}\text { Moyenne } \\
\text { arithmétique }\end{array}$ & 0,286 & 0,576 & 0,431 & 0,350 & $0, \mathbf{3 1 8}$ \\
\hline
\end{tabular}

\section{DISCUSSION}

La sélection envisagée ne s'effectue que dans une direction et avec une intensité décroissante, liée à la proportion décroissante d'individus ayant moins de douze tétines. En outre, les générations se chevauchent; dès lors, on ne se trouve pas dans les conditions d'une expérience de sélection permettant la meilleure estimation de l'héritabilité réalisée (FALCONER, I960; HILL, I97I, I972 $a$, I972 b). Néanmoins, on peut tenter de faire une estimation grossière de l'héritabilité réalisée sachant que, dans le matériel utilisé, l'intervalle de génération est de l'ordre de un an et demi.

La différentielle de sélection, à la génération $i$, est donnée par (HIL, I972 b) :

$$
s_{i}=Y_{t_{-1}}-\mathrm{X}_{t_{-1}}
$$

où $\mathrm{X}_{i}$ est la moyenne des individus mesurés à la génération $i$ et $\mathrm{Y}_{\mathfrak{l}}$, la moyenne des

Annales de Génétique animale. -1975. 
individus sélectionnés, appartenant à la même génération. La réponse qui résulte de cette sélection est donnée par (HILL, I972 b) :

$$
r_{i}=\mathrm{X}_{i}-\mathrm{X}_{i-1}
$$

Si on se rapporte au tableau $I$, on constate que la moyenne des pères des porcelets nés en 1963 est de 13,46 , alors que ces verrats appartiennent à la population née un an et demi plus tôt. A ce moment-là, la moyenne des mâles était (par interpolation) : $13,20+\mathbf{I} / 4(13,36-13,20)$ soit 13,24 . La différentielle de sélection pour les verrats est donc de $13,46-13,24=0,22$. On procède de même pour les truies et on trouve une différentielle de sélection de 0,195 . La différentielle moyenne pour les deux sexes est donc de 0,207 .

La réponse à cette sélection est égale à la moyenne en 1963 moins la moyenne un an et demi plus tôt, soit 0,1275 . On procède de même pour les années suivantes; on obtient ainsi cinq paires de valeurs : $s_{i}, r_{i}(i=\mathrm{I}$ à 5$)$. Chaque rapport $\boldsymbol{r}_{i} / s_{i}$ a la signification d'une héritabilité. La moyenne de ces cinq valeurs est de 0,44 . D'autre part, la régression de la réponse sur la différentielle de sélection $\left(b_{i}=\Sigma s_{i} r_{i} / \Sigma s_{i}^{2}\right.$, HILL, I972 $b$ ) est égale à 0,45 . On obtient ainsi deux estimations de l'héritabilité réalisée.

En fait, la seule sélection consciente réalisée consiste en l'élimination de sujets ayant moins de douze tétines. Cette seule sélection peut-elle expliquer la modification intervenue ? Le tableau 2 (dernière colonne) donne les pourcentages éliminés pour les six années envisagées ; chacun de ces pourcentages permet de calculer, si on admet une distribution gaussienne, une différentielle de sélection :

$$
s_{i}=i_{i} \cdot \sigma_{i}
$$

où $i_{i}$ est la différentielle de sélection en unités standard et $\sigma_{i}$ l'écart-type. Les différentielles de sélection calculées de cette façon sont systématiquement plus petites que celles obtenues par la première méthode. A des réponses équivalentes, il correspond donc des héritabilités réalisées plus élevées, voire supérieures à l'unité. Leur moyenne arithmétique est de 0,782 et la régression de la réponse sur la différentielle de sélection est égale à 0,84 . Ceci voudrait dire que l'élimination des sujets ayant moins de $\mathrm{I} 2$ tétines ne peut expliquer à elle seule l'évolution intervenue. Toutefois, cette façon de procéder suppose une distribution gaussienne. Une approche plus pragmatique consiste à calculer, d'après le tableau 2 et pour chaque année, la moyenne des porcelets ayant I 2 tétines et plus ; la différence entre cette moyenne et la moyenne générale correspondante est la différentielle de sélection $\left(s_{i}\right)$. Les valeurs ainsi obtenues pour les $s_{i}$ sont intermédiaires entre celles obtenues par les deux méthodes précédentes.

La moyenne des héritabilités obtenues est de o,60 et la régression de la réponse sur la différentielle est de 0,64 .

La conclusion reste inchangée : la seule élimination des sujets ayant moins de I 2 tétines n'explique pas le résultat obtenu. On peut se demander si une sélection consciente n'est pas venue s'ajouter à une première sélection automatique et ceci spécialement chez les verrats dont la moyenne est supérieure (sauf pour l'année 1965) à celle des truies (tabl. I).

Pour CAMERLYYNCK (I975), si cette sélection supplémentaire existe, « elle dépend 
plutôt de l'optique personnelle de l'éleveur que du point de vue officiel des dirigeants du livre généalogique ".

Remarquons en outre que le nombre de tétines paraît systématiquement plus élevé chez les mâles que chez les femelles. Celles-ci sont donc, en moyenne, plus proches du seuil d'élimination de douze tétines que les mâles. Néanmoins, la proportion d'individus ayant moins de douze tétines est plus grande chez les mâles que chez les femelles; on se serait attendu à l'inverse. Une variance moins grande du nombre de tétines chez les femelles aurait pu expliquer ce paradoxe, or la variance des femelles n'est pas plus petite que celle des mâles.

Reçu pour publication en mars 1975.

\section{REMERCIEMENTS}

Les auteurs remercient l'Ir R. CAMERLYNCK, ingénieur principal, qui a bien voulu leur fournir les données indispensables à la réalisaton de ce travail. Ils adressent également leurs remerciements à M. L. Ollivier (I. N. R. A.) dont les suggestions leur ont été très utiles.

\section{SUMMARY}

\section{EFFICIENCY OF A SELECTION CONCERNING \\ THE NIPPLE NUMBER IN PIETRAIN PIG}

The effects of a selection for an increased number of nipples in the Piétrain pig breed are studied for the period 1961-1971. The mean number of nipples has increased from 13,17 to 13,59 and the percentage of individuals culled for having less than 12 nipples, has decreased from 6,49 to $\mathrm{I}, 78 \mathrm{p}$. 100. Extreme phenotypes for the breed ( 17 and 18 nipples) have appeared at the end of the period considered.

\section{RÉFÉRENCES BIBLIOGRAPHIQUES}

CAMERLYNCK R., 1975. Communication personnelle.

Falconer D. S., 1960. Introduction to Quantitative Genetics. Ronald Press.

Hanset R., Camerlynck R., 1974. L'héritabilité du nombre de mamelles chez le Porc de Piétrain et chez le Porc Landrace Belge. Ann. Genét. Sel. Anim., 6, 9r-roz.

HIrL W. G., 197r. Design and efficiency of selection experiments for estimating genetic parameters. Biometrics, 27, 293-3II.

HrLL W. G., 1972 a. Estimation of realised heritabilities from selection experiments. I. Divergent selection. Biometrics, 28, 747-765.

HILE W. G., $1972 b$. Estimation of realised heritabilities from selection experiments. II. Selection in one direction. Biometrics, 28, 767-780. 